Homology, Homotopy and Applications, vol.4(1), 2002, pp.71-85

\title{
METRIZABLE SHAPE AND STRONG SHAPE EQUIVALENCES
}

\author{
L. STRAMACCIA \\ (communicated by Walter Tholen)
}

\begin{abstract}
In this paper we construct a functor $\Phi: \operatorname{pro} \mathcal{T} o p \rightarrow \operatorname{pro} \mathcal{A N} \mathcal{R}$ which extends Mardešić correspondence that assigns to every metrizable space its canonical $\mathcal{A N} \mathcal{N}$-resolution. Such a functor allows one to define the strong shape category of prospaces and, moreover, to define a class of spaces, called strongly fibered, that play for strong shape equivalences the role that $\mathcal{A N} \mathcal{N}$ spaces play for ordinary shape equivalences. In the last section we characterize SSDR-promaps, as defined by Dydak and Nowak, in terms of the strong homotopy extension property considered by the author.
\end{abstract}

\section{Introduction}

In ordinary Shape Theory there is a canonical way of associating with every topological space $X$ an inverse system $\check{\mathrm{X}}$ of absolute neighborhood retracts, namely its Čech system [15]. It is an inverse system in the homotopy category ho Top of topological spaces, whose bonding morphisms are homotopy classes of maps. This gives a functor ho $\mathcal{T}$ op $\rightarrow \operatorname{pro}($ ho $\mathcal{A N} \mathcal{N})$, where $\mathcal{A N \mathcal { R }}$ is the category of absolute neighborhood retracts. In Strong Shape Theory [14] one associates with every space $X$ an inverse system $\mathrm{X}$ in the category $\mathcal{T} o p$ of topological spaces, bonded by continu-

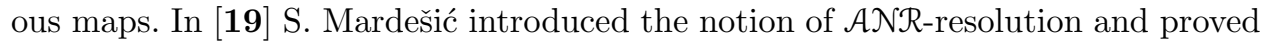

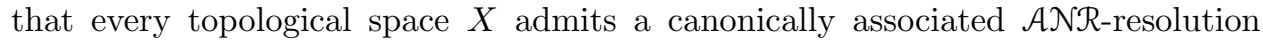
$M(X) \in \operatorname{pro} \mathcal{A} \mathcal{N} \mathcal{R}$. However, the correspondence $X \mapsto M(X)$ does not give a functor $\mathcal{T} o p \rightarrow \operatorname{pro} \mathcal{A} \mathcal{N} \mathcal{R}$. In their 1991 paper [8], Dydak and Nowak tried to overcome such difficulties defining a Mardešić-like functor $\mathcal{T}_{o p} \rightarrow \operatorname{pro} \mathcal{A N} \mathcal{R}$ but, due to some technical error, their construction there does not work (see [14], [9]). In another more recent paper [9], the same authors correct their errors adopting a different point of view. In this paper we undertake the program above and construct a functor $\Phi:$ proTop $\rightarrow$ pro $\mathcal{A} \mathcal{N} \mathcal{R}$, from the category of prospaces (inverse systems of topological spaces) to the category of inverse systems of absolute neighborhood retracts, which has the following properties:

Received January 25, 2002, revised August 23, 2002; published on September 9, 2002.

2000 Mathematics Subject Classification: 55P55, 55P05, 55P10, 18B30, 18G55, 55P60, 54B30.

Key words and phrases: Metrizable proreflector, shape equivalence, strong shape equivalence, double mapping cylinder, SSDR-promaps, strong homotopy extension property.

(C) 2002, L. Stramaccia. Permission to copy for private use granted. 
i) the restriction of $\Phi$ to the category of metrizable spaces coincides with Mardešić's correspondence which assigns to every space its canonical $\mathcal{A N} \mathcal{N}$-resolution $[\mathbf{1 3}]$,

ii) $\Phi$ has a reflective lifting ho $(\Phi):$ ho $(\operatorname{pro} \mathcal{T} o p) \rightarrow$ ho $(\operatorname{pro} \mathcal{A N \mathcal { N }})$ to the Steenrod homotopy categories,

iii) the restriction of $\mathrm{ho}(\Phi)$ to $\mathrm{ho}(\mathcal{T} o p)$ is naturally equivalent to Cathey and Segal's functor $R: \operatorname{ho}(\mathcal{T} o p) \rightarrow$ ho(pro $\mathcal{A N \mathcal { N } )}[\mathbf{5}]$,

iv) one can define the strong shape category of prospaces $s S h$ (proTop) as a natural extension of the category $s S h(\mathcal{T} o p)$, defined in [5], [19], considering the full image factorization of ho( $\Phi)$. It is shown that $s S h$ (proTop) can be obtained localizing proTop at the class of strong shape equivalences (cf.[17]).

Crucial for the definition of the functor $\Phi$ is the consideration of the metrizable

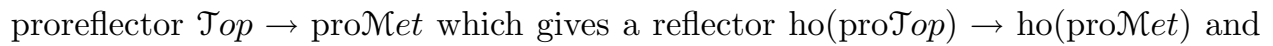
the fact that the strong shape theory of metrizable spaces is well settled in the literature.

The existence of the functor $\Phi$ allows one to characterize strong shape equivalences as those maps inducing bijections $f_{Z}^{*}:[Y, Z] \rightarrow[X, Z]$, between sets of homotopy classes, for every strongly fibered space $Z$ (section 2 ). Hence, strongly fibered spaces play, for strong shape equivalences, the role that ANR-spaces play for ordinary shape equivalences. Such a result was already stated by Dydak and Nowak in [8] and corrected in $[\mathbf{9}]$, where $\mathrm{SSDR}_{\mathcal{T}_{o p}}$ - fibrant spaces were introduced. We compare our results with those of [9] in last section. In particular we prove that SSDRpromaps of [9] coincide with the class of level cofibrations that are strong shape equivalences. As a fundamental tool we use a generalization of the SHEP (strong homotopy extension property), introduced in [21].

\section{Procategories and Localizations.}

Let $\mathcal{C}$ be any category. The category proC of inverse system in $\mathcal{C}$ has objects the contravariant functors $\mathrm{X}: \Lambda \rightarrow \mathcal{C}$, where $\Lambda=(\Lambda, \leqslant)$ is a directed set. An inverse system in $\mathcal{C}$ will be explicitly denoted by $\mathrm{X}=\left(X_{\lambda}, x_{\lambda \lambda^{\prime}}, \Lambda\right)$, where $X_{\lambda}=\mathrm{X}(\lambda)$ and $x_{\lambda \lambda^{\prime}}=\mathrm{X}\left(\lambda \leqslant \lambda^{\prime}\right)$.

We refer to [15] for all details concerning the definition of proe, but it will be useful to recall the following facts :

- a morphism $\mathrm{x}: X \rightarrow \mathrm{X}$, where $X \in \mathcal{C}$, is a family $\mathrm{x}=\left\{x_{\lambda}: X \rightarrow X_{\lambda} \mid \lambda \in \Lambda\right\}$ of morphisms of $\mathcal{C}$, with the property that $x_{\lambda \lambda^{\prime}} \circ x_{\lambda^{\prime}}=x_{\lambda}$, for all $\lambda \leqslant \lambda^{\prime}$.

- given a morphism $\mathrm{f}: \mathrm{X} \rightarrow \mathrm{Y}$ in proC, it is always possible to assume, up to isomorphisms, that $\Lambda$ is cofinite (that is: every $\lambda \in \Lambda$ has only finitely many predecessors), that $\mathrm{Y}=\left(Y_{\lambda}, q_{\lambda \lambda^{\prime}}, \Lambda\right)$ is indexed over the same directed set as $\mathrm{X}$ and that $f$ is a level morphism, that is given by a family $\left\{f_{\lambda}: X_{\lambda} \rightarrow Y_{\lambda} \mid \lambda \in \Lambda\right\}$ of morphisms of $\mathcal{C}$, with $y_{\lambda \lambda^{\prime}} \circ f_{\lambda^{\prime}}=f_{\lambda} \circ x_{\lambda \lambda^{\prime}}$, for $\lambda \leqslant \lambda^{\prime}$ ([15], Thm.3.1). Note that a level morphism is actually a natural transformation of functors.

1.1. A full subcategory $\mathcal{K}$ of $\mathcal{C}$ is proreflective in $\mathcal{C}([\mathbf{1 6}],[\mathbf{2 0}],[\mathbf{2 2}])$ if, for every $X \in \mathcal{C}$, there exists an inverse system $\mathrm{X} \in$ proK $\mathcal{K}$ and a morphism $\mathrm{x}: X \rightarrow \mathrm{X}$ in 
proe, which is universal (initial) with respect to every other morphism $f: X \rightarrow \mathrm{K}$, with $\mathrm{K} \in$ pro $\mathcal{K}$. In such a case $\mathrm{x}: X \rightarrow \mathrm{X}$ is called a $\mathcal{K}$-expansion for $X$. It is clear that a $\mathcal{K}$-expansion for $X$ is uniquely determined up to isomorphisms in pro $\mathcal{K}$. This fact allows one to define a functor $P: \mathcal{C} \rightarrow$ pro $\mathcal{K}, X \mapsto \mathrm{X}$, which is is called the proreflector.

Let $\mathcal{B}$ be any category having inverse limits. Every functor $F: \mathcal{C} \rightarrow \mathcal{B}$ has an extension $F^{*}: \operatorname{pro} \mathcal{C} \rightarrow \mathcal{B}$ which is defined by $F^{*}=\lim \cdot \operatorname{pro} F$ where $\operatorname{pro} F: \operatorname{proC} \rightarrow$ pro $\mathcal{B}$ is the functor such that $\operatorname{pro} F(\mathrm{X})=\left(F\left(X_{\lambda}\right), F\left(x_{\lambda \lambda^{\prime}}\right), \Lambda\right)$, while $\lim :$ pro $\mathcal{B} \rightarrow \mathcal{B}$ is the inverse limit functor. We give now a construction for the functor $F^{*}$ in the case $\mathcal{B}=$ pro $\mathcal{K}$, for some category $\mathcal{K}$.

Let $\mathrm{X}=\left(X_{\lambda}, x_{\lambda \lambda^{\prime}}, \Lambda\right) \in$ proC and let $F\left(X_{\lambda}\right)=\left(K_{i}^{\lambda}, k_{i i^{\prime}}^{\lambda}, I_{\lambda}\right)$, for every $\lambda \in \Lambda$. Then, $\left(F\left(X_{\lambda}\right), F\left(x_{\lambda \lambda^{\prime}}\right), \Lambda\right)$ is an inverse system in proK, whose inverse limit is the system

$$
F^{*}(\mathrm{X})=\left(K_{i}^{\lambda}, k_{i i^{\prime}}^{\lambda \lambda^{\prime}}, \Gamma\right),
$$

where $\Gamma=\bigcup\left\{\Lambda \times I_{\lambda} \mid \lambda \in \Lambda\right\}$ is directed by the relation

$$
(\lambda, i) \leqslant\left(\lambda^{\prime}, i^{\prime}\right) \Leftrightarrow\left\{\begin{array}{l}
\lambda \leqslant \lambda^{\prime} \text { in } \Lambda, \text { and } \\
k_{i i^{\prime}}^{\lambda \lambda^{\prime}}: K_{i^{\prime}}^{\lambda^{\prime}} \rightarrow K_{i}^{\lambda} \text { is part of } F\left(x_{\lambda \lambda^{\prime}}\right) .
\end{array}\right.
$$

Let $\mathrm{f}: \mathrm{X} \rightarrow \mathrm{Y}$ be a level morphism in proC, with $\mathrm{Y}=\left(Y_{\lambda}, y_{\lambda \lambda^{\prime}}, \Lambda\right)$ and $\mathrm{f}=\left\{f_{\lambda}\right.$ : $\left.X_{\lambda} \rightarrow Y_{\lambda} \mid \lambda \in \Lambda\right\}$. If we assume, as it is possible, that each $F\left(f_{\lambda}\right): F\left(X_{\lambda}\right) \rightarrow$ $F\left(Y_{\lambda}\right), \lambda \in \Lambda$, is a level morphism, then $F\left(Y_{\lambda}\right)=\left(H_{i}^{\lambda}, h_{i i^{\prime}}^{\lambda}, I_{\lambda}\right)$, hence it follows that

$$
F^{*}(\mathrm{Y})=\left(H_{i}^{\lambda}, h_{i i^{\prime}}^{\lambda \lambda^{\prime}}, \Gamma\right),
$$

while $F^{*}(\mathrm{f})$ is the level morphism given by

$$
F^{*}(\mathbf{f})=\left\{F\left(f_{\lambda}\right)_{i}: K_{i}^{\lambda} \rightarrow H_{i}^{\lambda} \mid(\lambda, i) \in \Gamma\right\} .
$$

Note that, if $P: \mathcal{C} \rightarrow$ pro $\mathcal{K}$ is a proreflector, then $P^{*}:$ proC $\rightarrow$ pro $\mathcal{K}$ is actually a reflector $[\mathbf{2 0}],[\mathbf{2 2}]$.

1.2. Recall that, given a class $\Sigma$ of morphisms in a category $\mathcal{C}$, the localization of $\mathcal{C}$ at $\Sigma$ is a pair $\left(\mathcal{C}\left[\Sigma^{-1}\right], L_{\Sigma}\right)$, where $\mathcal{C}\left[\Sigma^{-1}\right]$ is a category (possibly in a larger universe) having the same objects as $\mathcal{C}$ and $L_{\Sigma}: \mathcal{C} \rightarrow \mathcal{C}\left[\Sigma^{-1}\right]$ is a functor which is the identity on objects, having the following properties :

- $L_{\Sigma}$ inverts all morphisms of $\Sigma$, that is $L_{\Sigma}(s)$ is an isomorphism in $\mathcal{C}\left[\Sigma^{-1}\right]$, for all $s \in \Sigma$,

- $L_{\Sigma}$ is universal (initial) among all functors $F: \mathcal{C} \rightarrow \mathcal{E}$ that invert all morphisms of $\Sigma$.

$\Sigma$ is usually called the class of weak equivalences of $\mathcal{C}$.

If $\mathcal{D}$ is another category, endowed with a notion $\Delta$ of weak equivalences, then a functor $F: \mathcal{C} \rightarrow \mathcal{D}$ can be extended to a functor $\widetilde{F}: \mathcal{C}\left[\Sigma^{-1}\right] \rightarrow \mathcal{D}\left[\Delta^{-1}\right]$ if and only if $F$ preserves weak equivalences, that is $F(s) \in \Delta$, for all $s \in \Sigma . \widetilde{F}$ is the unique functor satisfying $\widetilde{F} \circ L_{\Delta}=L_{\Sigma} \circ F$; it acts on objects as $F$ does [18]. 
Let $\mathcal{C}=\mathcal{T}$ op be the category of topological spaces and let $\Sigma$ be the class of homotopy equivalences. Then, $\mathcal{T} o p\left[\Sigma^{-1}\right]=\mathrm{ho}(\mathcal{T} o p)$ is the usual homotopy category of spaces. In general, if $\mathcal{C}$ has a Quillen model structure, with $\Sigma$ the class of its weak equivalences, then hoC $=\mathcal{C}\left[\Sigma^{-1}\right]$. Moreover, proC inherits a Quillen model structure and its (Steenrod) homotopy category is ho(proe $)=$ proC $\left[\Sigma^{*-1}\right]$, where $\Sigma^{*}$ is the class of level weak equivalences, that is the class of those level morphisms which belong levelwise to $\Sigma$. $\Sigma^{*}$ will usually be considered as the class of weak equivalences of proC $[\mathbf{1 0}],[\mathbf{1 6}]$.

Theorem 1.3. (cf. [19]) Let $\mathcal{C}$ and $\mathcal{K}$ have classes of weak equivalences $\Sigma$ and $\Pi$, respectively, and let $P: \mathcal{C} \rightarrow$ pro $\mathcal{K}$ be any functor. If $P$ preserves weak equivalences, then also $P^{*}$ preserves weak equivalences. If, moreover, $P$ is a proreflector, then $\widetilde{P^{*}}:(\operatorname{proC})\left[\Sigma^{*-1}\right] \rightarrow(\operatorname{proC})\left[\Gamma^{*-1}\right]$ is a reflector.

Proof. Let $\mathrm{f} \in \Sigma^{*}, \mathrm{f}=\left\{f_{\lambda}\right\} . P^{*}(\mathrm{f})$ has level components of the form $P\left(f_{\lambda}\right)_{i},(\lambda, i) \in$ $\Gamma$, which are all members of $\Pi$, by assumption. If $P$ is a proreflector and $\Pi=$ $\Sigma \cap\{$ morphisms of $\mathcal{K}\}$, then $P^{*}$ is a reflector, hence left adjoint to the embedding $E:$ pro $\mathcal{K} \rightarrow$ proC. Since both $P^{*}$ and $E$ preserve weak equivalences, the assertion follows from ([2], Thm.1.1).

1.4. The usual cylinder functor on $\mathcal{T} o p$ can be extended naturally to a cylinder functor on proTop : for every $\mathrm{X}=\left(X_{\lambda}, x_{\lambda \lambda^{\prime}}, \Lambda\right) \in$ proTop, let $\mathrm{X} \times I=\left(X_{\lambda} \times\right.$ $\left.I, x_{\lambda \lambda^{\prime}} \times 1, \Lambda\right)$, where $I$ is the unit interval. One obtains, as a consequence, a notion of (global) homotopy between promaps (that is: between morphisms of prospaces) and a corresponding notion of (global) homotopy equivalence. Two promaps $\mathrm{f}, \mathrm{g}$ : $\mathrm{X} \rightarrow \mathrm{Y}$ are globally homotopic if there is a homotopy $\mathrm{H}: \mathrm{X} \times I \rightarrow \mathrm{Y}$ such that $\mathrm{H} \circ \mathrm{e}^{0}=\mathrm{f}$ and $\mathrm{H} \circ \mathrm{e}^{1}=\mathrm{g}$, where $\mathrm{e}^{0}, \mathrm{e}^{1}: \mathrm{X} \rightarrow \mathrm{X} \times I$ are the obvious promaps. The quotient category of proTop modulo global homotopy is denoted by $\pi$ (pro $\mathcal{T} o p$ ) and $\pi:$ proTop $\rightarrow \pi($ proTop $)$ is the quotient functor. In general, the classes of global and level homotopy equivalences in proTop do not coincide, as shown in ([10], pp.55-56); however, every global homotopy equivalence $\mathrm{X} \rightarrow \mathrm{Y}$ is a level homotopy equivalence, whenever the bonding morphisms of $\mathrm{X}$ are epi ([19], Cor. 1.3).

1.5. Let $F: \mathcal{C} \rightarrow \mathcal{K}$ be any functor and let $\mathcal{C}_{F}$ be the category having the same objects as $\mathcal{C}$ while a morphism in $\mathcal{C}_{F}(X, Y)$ is a triple $\left(1_{X}, u, 1_{Y}\right)$, where $u \in \mathcal{K}(F(X), F(Y)) . \mathcal{C}_{F}$ is called the full image of $F$. There are functors $F^{0}: \mathcal{C} \rightarrow$ $\mathcal{C}_{F}$ and $F^{1}: \mathcal{C}_{F} \rightarrow \mathcal{K}$, defined by $F^{0}(X)=X$ and $F^{0}(f)=\left(1_{X}, F(f), 1_{Y}\right)$, for $f: X \rightarrow Y$ in $\mathcal{C}$, and $F^{1}(X)=F(X), F^{1}\left(1_{X}, u, 1_{Y}\right)=u$. They give a factorization $F=F^{1} \circ F^{0}$ of $F$ which is uniquely determined, up to an isomorphism, among all factorizations $F=H^{\prime \prime} \circ H^{\prime}$, where $H^{\prime}$ is bijective on objects and $H^{\prime \prime}$ is fully faithful. $F=F^{1} \circ F^{0}$ is called the full image factorization of $F[\mathbf{1 8}]$. Recall that, when $F$ is a reflector and $\Sigma_{F}$ is the class of morphisms of $\mathcal{C}$ inverted by $F$, then there is an isomorphism $\mathcal{K} \cong \mathcal{C}\left[\Sigma_{F}^{-1}\right]([\mathbf{1 8}], 19.3 .1)$. Moreover, from the uniqueness of the full image factorization and since $L_{\Sigma_{F}}$ is the identity on objects, one also obtains an isomorphism $\mathcal{C}\left[\Sigma_{F}^{-1}\right] \cong \mathcal{C}_{F}$. Let $\Sigma_{F^{0}}$ denote the class of morphisms in $\mathcal{C}$ that are inverted by $F^{0}$. Then clearly $\Sigma_{F}=\Sigma_{F^{0}}$ holds.

In what follows we give a brief account of the construction of the Steenrod homotopy category ho(proTop) of proTop, following the point of view of [5]. 
Definition 1.6. Let $f: X \rightarrow Y$ and $p: E \rightarrow B$ be promaps. $f$ has the left lifting property with respect to $p$ (and $p$ has the right lifting property with respect to $f$ ) if every commutative square

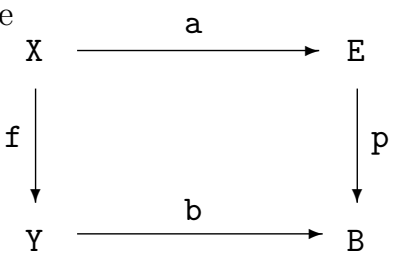

has a filler $\mathrm{h}: \mathrm{Y} \rightarrow \mathrm{E}$, such that $\mathrm{h} \circ \mathrm{f}=\mathrm{a}$ and $\mathrm{p} \circ \mathrm{h}=\mathrm{b}$.

Let $\Sigma$ be a a class of morphisms in proTop, then

- a promap p : E $\rightarrow$ B is a $\Sigma$-fibration if it has the right lifting property with respect to all $\mathrm{f} \in \Sigma$,

- a prospace $\mathrm{Z}=\left(Z_{\mu}, z_{\mu \mu^{\prime}}, M\right)$ is $\Sigma$-fibrant iff the unique morphism $\mathrm{Z} \rightarrow *$ is a $\Sigma$-fibration, where $*$ denotes the final object in $\mathcal{T}_{o p}$,

- a $\Sigma$-fibrant prospace $\mathrm{Z}$ is said to be strongly $\Sigma$-fibrant if, moreover, for every $\mu^{*} \in M$, the unique map $z_{\mu^{*}}: Z_{\mu^{*}} \rightarrow \lim _{\mu<\mu^{*}} Z_{\mu}$, induced by the bonding maps of the system, is a $\Sigma$-fibration,

- a topological space $Z$ is $\Sigma$-strongly fibered if it is the inverse limit of a strongly $\Sigma$-fibrant prospace $\mathrm{Z} \in \operatorname{pro} \mathcal{A} \mathcal{N} \mathcal{R}$.

In the homotopy theory of proTop, as defined in [10], a promap $\mathrm{f}$ is a trivial cofibration if it has the left lifting property with respect to every Hurewicz fibration $p: E \rightarrow B$ in $\mathcal{T} o p$. This notion is a natural extension of that of trivial cofibration in $\mathcal{T}$ op. On the other hand, it is clear that a map $p$ having the right lifting property with respect to all trivial cofibrations $f$ in pro $\mathcal{T} o p$, has to be a Hurewicz fibration. In the sequel, for $\Sigma$ the class of trivial cofibrations in proTop, we shall speak of (strongly) fibrant prospaces and strongly fibered spaces, omitting the reference to the class $\Sigma$.

1.7. There is a reflective functor $F: \pi($ proTop $) \rightarrow \pi(\text { proTop })_{f}$ onto the full subcategory of fibrant prospaces [5], with unit of adjunction $\left[i_{\mathrm{X}}\right]: \mathrm{X} \rightarrow \widehat{\mathrm{X}}$, where $i_{\mathrm{X}}$ is a trivial cofibration. By $([\mathbf{5}]$, Prop. 3.3) $F$ has a reflective restriction $F: \pi(\operatorname{pro} \mathcal{A N} \mathcal{N}) \rightarrow$ $\pi(\operatorname{pro} \mathcal{A N} \mathcal{N})_{s f}$, where $\pi(\operatorname{pro} \mathcal{A N \mathcal { N }})_{s f}$ is the full subcategory of strongly fibrant prospaces. For $\mathrm{Z} \in \operatorname{pro} \mathcal{A N \mathcal { R }}, \mathrm{i}_{\mathrm{Z}}: \mathrm{Z} \rightarrow \widehat{\mathrm{Z}}$ is called the strongly fibrant modification of $\mathrm{Z}$.

ho(proTop) is the full image of the functor $F$ above and is equipped with the canon-

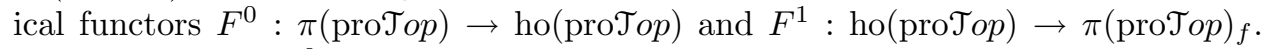
The functor $L=F^{0} \circ \pi:$ proTop $\rightarrow$ ho(pro $\left.\mathcal{T}_{o p}\right)$ is known to localize proTop at the class of trivial cofibrations and also at the class of level homotopy equivalences [10], $[16]$.

Remark 1.8. For $\mathrm{X}, \mathrm{Y} \in$ proTop, with $\mathrm{Y}$ fibrant, there is a natural bijection

$$
\operatorname{ho}(\operatorname{pro} \mathcal{T} o p)(\mathrm{X}, \mathrm{Y}) \cong[\mathrm{X}, \mathrm{Y}],
$$

where $[\mathrm{X}, \mathrm{Y}]$ is the set of global homotopy classes of morphisms $\mathrm{X} \rightarrow \mathrm{Y}$. This is because every prospace $\mathrm{X}$ is in fact cofibrant in proTop ([10], Prop.3.4.1, pag. 95). 


\section{The functor $\Phi: \operatorname{pro} \mathcal{T} o p \rightarrow \operatorname{pro} \mathcal{A} \mathcal{N} \mathcal{R}$ and the category $s S h($ proTop $)$.}

The category $\mathcal{M}$ et of metrizable spaces is proreflective in $\mathcal{T} o p$. In order to obtain the metrizable expansion $\mathrm{x}: X \rightarrow \mathrm{X}$ of a topological space $(X, \tau)$, let us consider the set $\Lambda$ of all continuous pseudometrics on $X$, directed by the relation

$$
\lambda \leqslant \lambda^{\prime} \Longleftrightarrow \tau_{\lambda} \subset \tau_{\lambda^{\prime}},
$$

Here $\tau_{\lambda}$ denotes the topology induced on $X$ by the pseudometric $\lambda$, while the continuity of $\lambda$ means that $\tau_{\lambda} \subset \tau[\mathbf{1}]$. Let $X_{\lambda}$ denote the metric identification of $\left(X, \tau_{\lambda}\right)$. For every $\lambda \in \Lambda$, let $x_{\lambda}: X \rightarrow X_{\lambda}$ be the identity map $(X, \tau) \rightarrow\left(X, \tau_{\lambda}\right)$ followed by the quotient map $\left(X, \tau_{\lambda}\right) \rightarrow X_{\lambda}$. Moreover, for $\lambda \leqslant \lambda^{\prime}$, let $x_{\lambda \lambda^{\prime}}: X_{\lambda^{\prime}} \rightarrow X_{\lambda}$ be the unique map induced on the quotients by the identity $\left(X, \tau_{\lambda^{\prime}}\right) \rightarrow\left(X, \tau_{\lambda}\right)$. We note explicitly that in the inverse system $\mathrm{X}=\left(X_{\lambda}, x_{\lambda \lambda^{\prime}}, \Lambda\right)$, the bonding morphisms $x_{\lambda \lambda^{\prime}}$ are all surjective maps. We shall denote by $P_{M}: \mathcal{T}_{o p} \rightarrow$ proMet, $X \mapsto \mathrm{X}$, the metrizable proreflector.

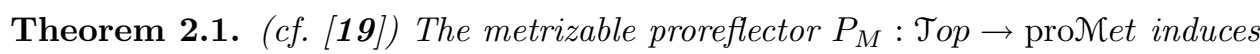
a reflector $\mathrm{ho}\left(P_{M}^{*}\right):$ ho(proTop $) \rightarrow$ ho(proNet $)$.

Proof. In view of Thm.1.3, it suffices to prove that $P_{M}$ preserves weak equivalences. Let us note that $P_{M}$ respects the cylinders, in the sense that, if $\mathrm{x}: X \rightarrow \mathrm{X}=$ $\left(X_{\lambda}, x_{\lambda \lambda^{\prime}}, \Lambda\right)$ is the metrizable expansion of the space $X$, then $\mathrm{x} \times 1: X \times I \rightarrow$ $\mathrm{X} \times I=\left(X_{\lambda} \times I, x_{\lambda \lambda^{\prime}} \times 1, \Lambda\right)$ is the metrizable expansion of $X \times I$, see ([19], Thm.2.3). It follows that $P_{M}$ takes homotopy equivalences to global homotopy equivalences. Since $\mathrm{X}$ has epi bonding morphisms, the proof is complete. The reflection morphism $\chi: \mathrm{X} \rightarrow P_{M}^{*}(\mathrm{X})$, for the prospace $\mathrm{X}$, is induced by the family $\left\{\mathrm{x}_{\lambda}: X_{\lambda} \rightarrow \mathrm{X}_{\lambda} \mid \lambda \in \Lambda\right\}$ of the metrizable expansions of each $X_{\lambda}$, following the construction given in the previous section (1.1).

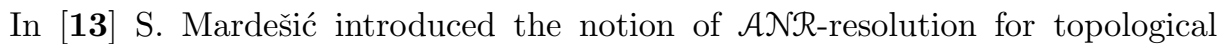
spaces and proved that every space $X$ has a canonically associated $\mathcal{A} \mathcal{N} \mathcal{R}$-resolution $\mathrm{m}_{X}: X \rightarrow M(X)$. Although the correspondence $\mathcal{T}_{o p} \rightarrow \operatorname{pro} \mathcal{A} \mathcal{N} \mathcal{R}, X \mapsto M(X)$, is not functorial in general, Cathey and Segal [5] proved that it induces a reflective functor between the Steenrod homotopy categories $R: \operatorname{ho}(\mathcal{T} o p) \rightarrow \operatorname{ho}(\operatorname{pro} \mathcal{A N} \mathcal{R})$. Moreover, they obtained the strong shape category $s S h(\mathcal{T} o p)$ and the strong shape functor $s S_{\mathcal{T}_{o p}}$ by taking the full image factorization of $R$ :

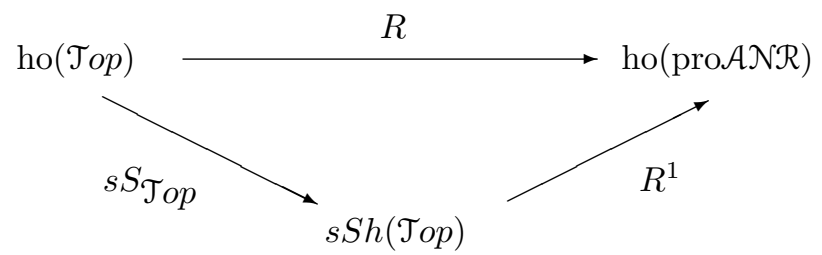

where $s S_{\mathcal{T} o p}=R^{0}$ is the identity on objects, while $R^{1}$ is fully faithful.

The fact that $R$ is a reflective functor means that, for every $X \in \mathcal{T} o p$ and for

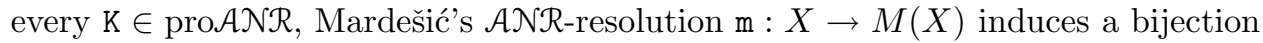
$\operatorname{ho}(\operatorname{proT} o p)(X, \mathrm{~K}) \cong \operatorname{ho}(\operatorname{pro} \mathcal{A} \mathcal{N} \mathcal{R})(M(X), \mathrm{K})$. 
Another feature of Mardešić's correspondence is that it becomes a functor

$$
M: \mathcal{M} e t \rightarrow \operatorname{pro} \mathcal{A N} \mathcal{N}
$$

when restricted to the category $\mathcal{M}$ et of metrizable spaces. This fact was pointed out in [19] and used to give an alternative description of the strong shape category of topological spaces. The same paper (Thm. 2.4) also gave a particularly simple construction for the $\mathcal{A} \mathcal{N} \mathcal{R}$-resolution $\mathrm{m}_{X}: X \rightarrow M(X)$ of a metrizable space $X$,

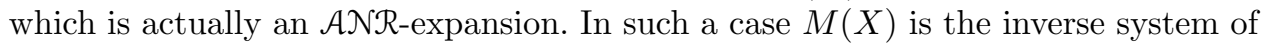
all open neighborhoods of $X$ in its convex hull $H(X)$ in the Banach space $C(X)$ of all real, bounded, continuous functions on $X$, while $\mathrm{m}_{X}$ is formed by all the inclusions.

Let us note that, lifting the functor $M: \mathcal{N} e t \rightarrow \operatorname{pro} \mathcal{A N} \mathcal{R}$ to the Steenrod homotopy categories, amounts to taking the restriction $\mathrm{ho}(M): \mathrm{ho}(\mathcal{M} e t) \rightarrow \operatorname{ho}(\operatorname{pro} \mathcal{A N} \mathcal{N})$ of Cathey and Segal's functor $R$, to the homotopy subcategory of metrizable spaces. It follows that $M: \mathcal{N} e t \rightarrow \operatorname{pro} \mathcal{A N} \mathcal{N}$ has to preserve weak equivalences. By Thm.1.1, the functor $M^{*}:$ proNet $\rightarrow \operatorname{pro} \mathcal{A} \mathcal{N} \mathcal{R}$ also preserves weak equivalences and has a lifting

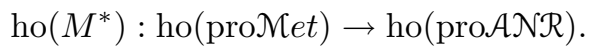

Theorem 2.2. ho $\left(M^{*}\right)$ is a reflector.

Proof. Let $\mathrm{X}=\left(X_{\lambda}, x_{\lambda \lambda^{\prime}}, \Lambda\right)$ be an inverse system of metrizable spaces. The family

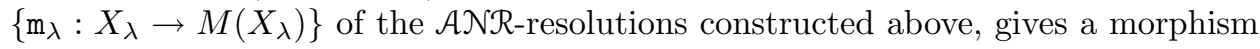

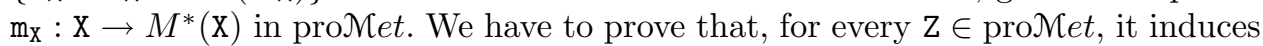
a bijection

$$
\mathrm{ho}(\operatorname{pro\mathcal {M}et})(\mathrm{x}, \mathrm{z}) \cong \operatorname{ho}(\operatorname{pro} \mathcal{A N \mathcal { R }})\left(M^{*}(\mathrm{x}), \mathrm{z}\right) \text {. }
$$

Let $i_{z}: Z \rightarrow \widehat{Z}$ be the strongly fibrant modification of $\mathrm{Z}$. By the preceding re-

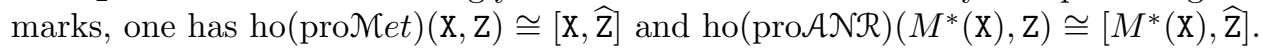
It follows that proving the formula above amounts to proving that $\mathrm{m}_{\mathrm{X}}$ induces a bijection

$$
\left[M^{*}(\mathrm{X}), \widehat{\mathrm{Z}}\right] \cong[\mathrm{X}, \widehat{\mathrm{Z}}] .
$$

This is a consequence of the fact that ho $(M)$ is reflective and of the construction of $M^{*}(\mathrm{X})$, as recalled in the first section.

Let us now define the functor

$$
\Phi: \operatorname{pro} \mathcal{T} o p \rightarrow \operatorname{pro} \mathcal{A N} \mathcal{R}
$$

as follows: for every $\mathrm{X} \in \operatorname{pro} \mathcal{T} o p$, let $\Phi(\mathrm{X})=M^{*}\left(P_{M}^{*}(\mathrm{X})\right)$. It is clear that ho( $\left.\Phi\right)$ : ho $(\operatorname{pro} \mathcal{T} o p) \rightarrow$ ho $(\operatorname{pro} \mathcal{A N} \mathcal{N})$ exists and can be written as ho $(\Phi)=$ ho $\left(M^{*}\right) \circ \mathrm{ho}\left(P_{M}^{*}\right)$. By (1.7) we may assume, without restriction of generality, that $\Phi(\mathrm{X})$ is strongly fibrant in pro $\mathcal{A N \mathcal { N }}$. Moreover, by the results above, it follows that ho $(\Phi)$ is a reflector. If $\mathrm{X}=\left(X_{\lambda}, x_{\lambda \lambda^{\prime}}, \Lambda\right)$, the reflection morphism $\mu: \mathrm{X} \rightarrow \Phi(\mathrm{X})$ is the promap obtained as the composition of $\chi: \mathrm{X} \rightarrow P_{M}^{*}(\mathrm{x}), \mathrm{m}_{P_{M}^{*}(\mathrm{x})}: P_{M}^{*}(\mathrm{x}) \rightarrow M^{*}\left(P_{M}^{*}(\mathrm{x})\right)$ and the strongly fibrant modification of $M^{*}\left(P_{M}^{*}(\mathrm{x})\right)$.

The restriction of ho( $\Phi)$ to ho( $\mathcal{T} o p)$ coincides with the functor $R$ of Cathey and 
Segal [19] and, consequently, it defines the same strong shape category for the class of topological spaces. The functor $\Phi$ is an extension of Mardešic 's functor defined on the subcategory of metrizable spaces. Let us define the strong shape category $s S h($ proTop) for inverse systems of topological spaces and the related strong shape functor $s S$, by taking the full image factorization of ho $(\Phi)$, as illustrated in the commutative diagram

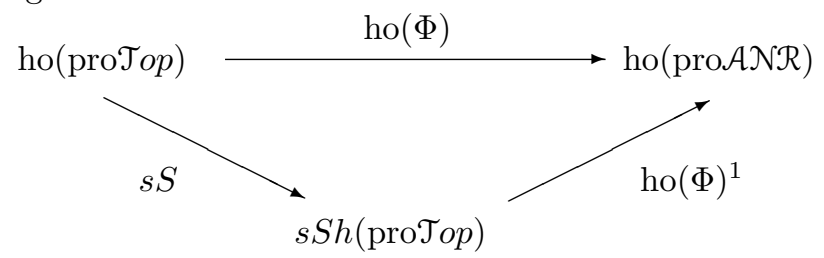

\section{Shape and Strong Shape Equivalences.}

A continuous map $f: X \rightarrow Y$ is said to be a (strong) shape equivalence if it becomes an isomorphism in the (strong) shape category of topological spaces, that is $s S(L(f))$ is an isomorphism in $s S h$ (proTop). We refer to [15] and [14] for basic facts concerning shape and strong shape theory. In particular we recall that:

- $f$ is a shape equivalence iff it induces a bijection $f_{K}^{*}:[Y, A] \rightarrow[X, A]$ between sets of homotopy classes, for all $A \in \mathcal{A N} \mathcal{N}$,

- a shape equivalence $f$ is a strong shape equivalence iff, given maps $g, h: Y \rightarrow$ $A, A \in \mathcal{A N \mathcal { R }}$, and a homotopy $F: X \times I \rightarrow A$ connecting $g \circ f$ and $h \circ f$, there exists a homotopy $G: Y \times I \rightarrow A$ connecting $g$ and $h$, such that $G \circ(f \times 1)$ is homotopic to $F$ w.r.t. end maps.

The notion of strong shape equivalence in proTop is the obvious generalization of the notion given previously $[\mathbf{8}],[\mathbf{1 4}]: \mathrm{f}: \mathrm{X} \rightarrow \mathrm{Y}$ is a strong shape equivalence whenever the following two conditions hold :

(SSE1) for every $A \in \mathcal{A N \mathcal { R }}$ and for every $\mathrm{h}: \mathrm{X} \rightarrow A$, there is a morphism $\mathrm{g}: \mathrm{Y} \rightarrow A$, such that $\mathrm{g} \circ \mathrm{f} \simeq \mathrm{h}$,

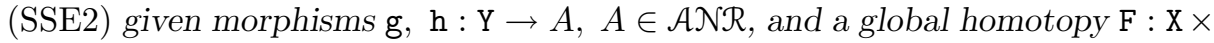
$I \rightarrow A$ joining $\mathrm{f} \circ \mathrm{g}$ and $\mathrm{f} \circ \mathrm{h}$, there exists a global homotopy $\mathrm{G}: \mathrm{Y} \times I \rightarrow A$ joining $\mathrm{g}$ and $\mathrm{h}$, such that $\mathrm{F}$ is homotopic to $\mathrm{G} \circ(\mathrm{f} \times 1)$ w.r.t. end morphisms.

Notice that, if a composition gof satisfies (SSE1), then $\mathrm{f}$ satisfies (SSE1). In fact, that $\mathrm{f}$ satisfies (SSE1) amounts to saying that the induced map $\mathrm{f}^{*}:[\mathrm{Y}, A] \rightarrow[\mathrm{X}, A]$ is onto. On the other hand one has $(g \circ f)^{*}=f^{*} \circ g^{*}$.

Theorem 3.1. The morphism $\mu: \mathrm{X} \rightarrow \Phi(\mathrm{X})$ is a strong shape equivalence.

Proof. This is almost obvious. $\operatorname{ho}(\Phi)(\mu)$ must be an isomorphism in ho(pro $\mathcal{A N R}$ ), because of the reflectivity. Since $\mathrm{ho}(\Phi)=\mathrm{ho}(\Phi)^{1} \circ s S$ and $\mathrm{ho}(\Phi)^{1}$ is fully faithful, it follows that $s S(\mu)$ is an isomorphism in the strong shape category $s S h$ (pro $\mathcal{T} o p$ ), hence $\mu$ is a strong shape equivalence. 
Corollary 3.2. For prospaces $\mathrm{X}, \mathrm{Y}$, the following relation holds

$$
\operatorname{ho}(\operatorname{proTop})(\mathrm{X}, \mathrm{Y}) \cong[\Phi(\mathrm{X}), \Phi(\mathrm{Y})] \text {. }
$$

We need to consider now the following facts. Let $\mathrm{f}: \mathrm{X} \rightarrow \mathrm{Y}, \mathrm{f}=\left\{f_{\lambda} \mid \lambda \in \Lambda\right\}$, be a level promap.

3.3. For every $\lambda \in \Lambda$, let $M\left(f_{\lambda}\right)$ be the mapping cylinder of $f_{\lambda}[\mathbf{1 2}]$, with canonical maps $\Pi_{\lambda}: X_{\lambda} \times I \rightarrow M\left(f_{\lambda}\right)$ and $j_{\lambda}: Y_{\lambda} \rightarrow M\left(f_{\lambda}\right)$, such that $\Pi_{\lambda} \circ e_{0, \lambda}=j_{\lambda} \circ f_{\lambda}$. Note that $j_{\lambda}$ has a left inverse $p_{\lambda}$, such that $p_{\lambda} \circ \Pi_{\lambda}=f_{\lambda} \circ \sigma_{\lambda}$, where $\sigma_{\lambda}: X_{\lambda} \times I \rightarrow X_{\lambda}$ is the usual map. Then $f_{\lambda}$ has a decomposition $f_{\lambda}=f_{\lambda}^{1} \circ f_{\lambda}^{0}$, where $f_{\lambda}^{0}: X_{\lambda} \rightarrow M\left(f_{\lambda}\right)$ is a cofibration and $f_{\lambda}^{1}: M\left(f_{\lambda}\right) \rightarrow Y_{\lambda}$ is a homotopy equivalence. Since such a decomposition is functorial [11], one can define (levelwise) the mapping cylinder decompositon of the promap $f$, given by

$$
\mathrm{X} \stackrel{\mathrm{f}}{\longrightarrow} \mathrm{Y}=\mathrm{X} \stackrel{\mathrm{f}^{0}}{\longrightarrow} M(\mathrm{f}) \stackrel{\mathrm{f}^{1}}{\longrightarrow} \mathrm{Y}
$$

where $\mathrm{f}^{0}=\left\{f_{\lambda}^{0} \mid \lambda \in \Lambda\right\}$ is a level cofibration, $\mathrm{f}^{1}=\left\{f_{\lambda}^{1} \mid \lambda \in \Lambda\right\}$ is a level homotopy equivalence and $M(\mathrm{f})=\left(M\left(f_{\lambda}\right), m_{\lambda \lambda^{\prime}}, \Lambda\right)$. The maps $m_{\lambda \lambda^{\prime}}$ are obtained from the universal properties of the various mapping cylinders.

3.4. $D M\left(f_{\lambda}\right)$ denote the double mapping cylinder of $f_{\lambda}[\mathbf{1 2}],[\mathbf{1 4}],[\mathbf{2 1}]$, that is the adjunction space $\left(X_{\lambda} \times I\right) \cup_{f_{\lambda}}\left(Y_{\lambda} \times \partial I\right)$, equipped with canonical maps $K_{\lambda}: X_{\lambda} \times I \rightarrow$ $D M\left(f_{\lambda}\right)$ and $j_{i, \lambda}: Y_{\lambda} \rightarrow D M\left(f_{\lambda}\right), i=0,1$, such that $K_{\lambda} \circ e_{i}=j_{i, \lambda} \circ f_{\lambda}, i=0,1$. Since $D M\left(f_{\lambda}\right)$ is a colimit object, there is a unique map $V_{\lambda}: D M\left(f_{\lambda}\right) \rightarrow Y_{\lambda} \times I$, with the property that $V_{\lambda} \circ K_{\lambda}=f_{\lambda} \times 1$ and $V_{\lambda} \circ j_{i, \lambda}=e_{i}, i=0,1$. For every $\lambda \leqslant \lambda^{\prime}$, there is a unique map $n_{\lambda \lambda^{\prime}}: D M\left(f_{\lambda}^{\prime}\right) \rightarrow D M\left(f_{\lambda}\right)$, such that $n_{\lambda \lambda^{\prime}} \circ K_{\lambda^{\prime}}=K_{\lambda} \circ\left(x_{\lambda \lambda^{\prime}} \times 1\right)$ and $n_{\lambda \lambda^{\prime}} \circ j_{i, \lambda^{\prime}}=j_{i, \lambda} \circ y_{\lambda \lambda^{\prime}}, i=0,1$. The situation is better illustrated by the following commutative diagram

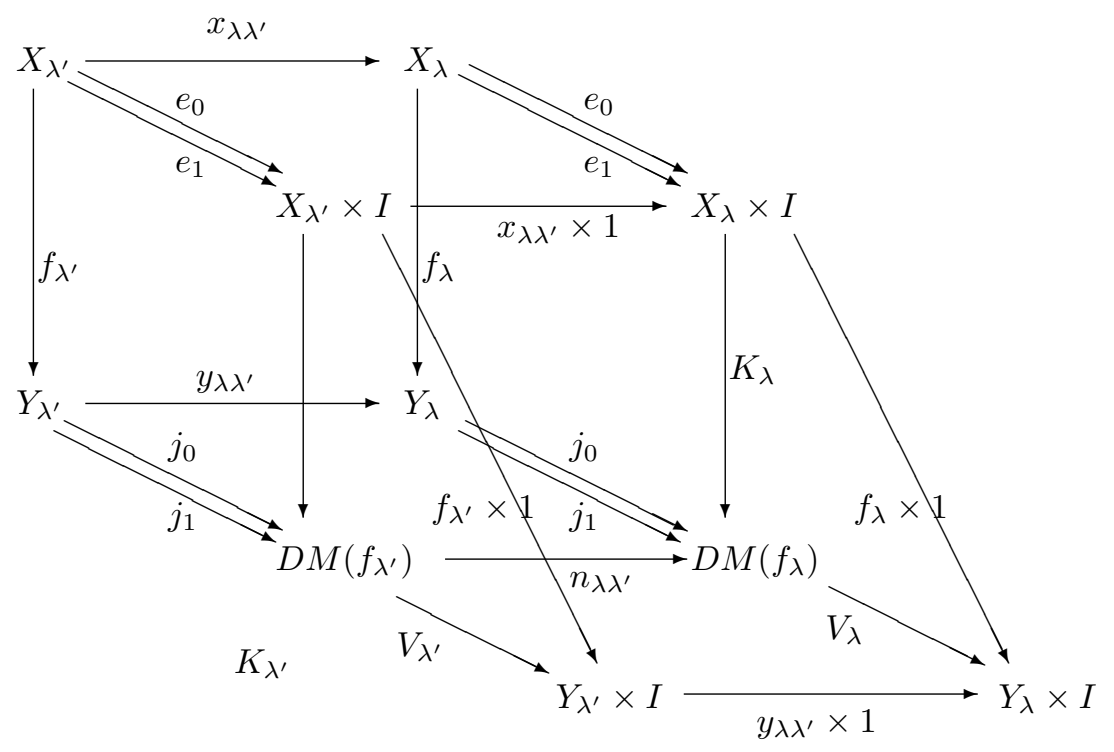


with the obvious meaning of the maps involved. It follows that there is an inverse system $D M(\mathrm{f})=\left(D M\left(f_{\lambda}\right), n_{\lambda \lambda^{\prime}}, \Lambda\right)$ and level maps $\mathrm{K}: \mathrm{X} \times I \rightarrow D M(\mathrm{f}), \mathrm{j}_{0}, \mathrm{j}_{1}$ : $\mathrm{Y} \rightarrow D M(\mathrm{f})$ and $\mathrm{V}: D M(\mathrm{f}) \rightarrow \mathrm{Y} \times I$, with $\mathrm{K} \circ \mathrm{e}_{i}=\mathrm{j}_{i} \circ \mathrm{f}, i=0,1$, and such that $\mathrm{f} \times 1=\mathrm{K} \circ \mathrm{V}$ and $\mathrm{V} \circ \mathrm{j}_{i}=\mathrm{e}_{i}, i=0,1$.

We point out that, if $\mathbf{f}$ is a level cofibration, then $\mathrm{V}$ is one too $[\mathbf{1 2}]$.

Theorem 3.5. The class of strong shape equivalences of proTop has the following properties:

1. contains all level homotopy equivalences,

2. if two of $\mathrm{f}, \mathrm{g}, \mathrm{g} \circ \mathrm{f}$ are strong shape equivalences, so is the third,

3. a level promap $\mathrm{f}$ is a strong shape equivalence iff $\mathrm{f}^{0}$ is,

4. if $\mathrm{f}$ is a strong shape equivalence and a level cofibration, then for every $\mathrm{g}: \mathrm{X} \rightarrow$ $A, A \in \mathcal{A} \mathcal{N} \mathcal{R}$, there is an $\mathrm{h}: \mathrm{Y} \rightarrow A$ such that $\mathrm{h} \circ \mathrm{f}=\mathrm{g}$.

Proof. (1) is clear (see also [8], 4.1, 4.2). (2) depends on the fact that $S s(\mathrm{~g} \circ \mathrm{f})=$ $S s(\mathrm{~g}) \circ S s(\mathrm{f})$. (3) follows from (2). (4) Since $\mathrm{f}$ is a level cofibration, there is a weak pushout diagram in pro $\mathcal{T} o p$, with respect to $\mathcal{A N \mathcal { R }}$

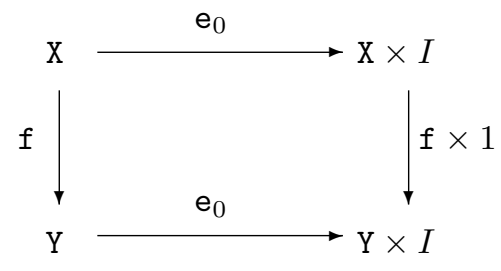

Given now promaps $\phi: \mathrm{Y} \rightarrow A, A \in \mathcal{A} \mathcal{N} \mathcal{R}$, and $\mathrm{F}: \mathrm{X} \times I \rightarrow A$ such that $\mathrm{F} \circ \mathrm{e}_{0}^{X}=$ $\phi \circ \mathrm{f}$, there exists a $\lambda \in \Lambda$, such that the relative $\lambda$-diagram commutes. Therefore there is a homotopy $G_{\lambda}: Y_{\lambda} \times I \rightarrow A$ with $G_{\lambda} \circ\left(f_{\lambda} \times 1\right)=F_{\lambda}$ and $G_{\lambda} \circ e_{0, \lambda}=\phi_{\lambda}$. Such data define a homotopy $\mathrm{G}: \mathrm{Y} \times I \rightarrow A$ with $\mathrm{G} \circ(f \times 1)=\mathrm{F}$ and $\mathrm{G} \circ \mathrm{e}_{0}^{Y}=\phi$. At this point the assertion follows from ([12], 2.2.4).

In view of the theorem above, one can restrict the study of strong shape equivalences to those promaps that are level cofibrations.

In [21] the strong homotopy extension property (SHEP) for maps has been introduced, with respect to $\mathcal{A N \mathcal { N }}$. This can be generalized to promaps in the following way: a promap $\mathrm{f}: \mathrm{X} \rightarrow \mathrm{Y}$ has the SHEP, w.r.t. $\mathcal{A} \mathcal{N} \mathcal{R}$, iff the following diagram

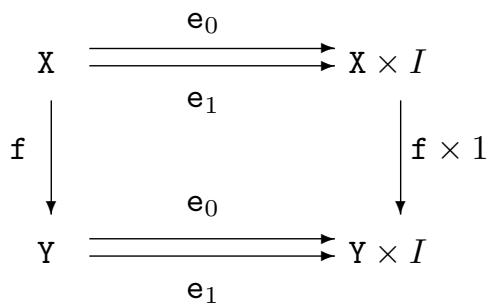

is a weak colimit in pro $\mathcal{T} o p$, w.r.t. $\mathcal{A} \mathcal{N} \mathcal{R}$. This means that, for given promaps $\mathrm{u}, \mathrm{v}$ : $\mathrm{Y} \rightarrow A$ and homotopy $\mathrm{H}: \mathrm{X} \times I \rightarrow A, A \in \mathcal{A} \mathcal{N} \mathcal{R}$, connecting $\mathrm{u} \circ \mathrm{f}$ and $\mathrm{v} \circ \mathrm{f}$, there exists a homotopy $\mathrm{G}: \mathrm{Y} \times I \rightarrow A$, connecting $\mathrm{u}$ and $\mathrm{v}$ and such that $\mathrm{H}=\mathrm{G} \circ(\mathrm{f} \times 1)$. 
Theorem 3.6. (cf. [21], sec.2) Let $\mathrm{f}: \mathrm{X} \rightarrow \mathrm{Y}$ be a level cofibration in proTop having property (SSE1). The following are equivalent :

1. $\mathrm{f}$ is a strong shape equivalence,

2. f has the SHEP w.r.t. ANNR,

3. $\mathrm{V}$ has property (SSE1).

Proof. (1) implies (2) : since $\mathrm{f}$ is a level cofibration, this follows from ([3], 7.2.5).

(2) implies (3) : Let $\alpha: D M(\mathrm{f}) \rightarrow A, A \in \mathcal{A N \mathcal { N }}$, and consider $\alpha \circ \mathrm{K}: \mathrm{X} \times I \rightarrow A$. It is a homotopy connecting $\alpha \circ j_{0} \circ f$ to $\alpha \circ j_{1} \circ f$, then there is a homotopy $\mathrm{T}: \mathrm{Y} \times I \rightarrow A$ such that $\mathrm{T} \circ(\mathrm{f} \times 1)=\alpha \circ \mathrm{K}$ and $\mathrm{T} \circ \mathrm{e}_{i}=\alpha \circ \mathrm{j}_{i}$. It follows that $\mathrm{T} \circ \mathrm{V} \circ \mathrm{K}=\mathrm{T} \circ(\mathbf{f} \times 1)=\alpha \circ \mathrm{K}$ and $\mathrm{T} \circ \mathrm{V} \circ \mathrm{j}_{i}=\mathrm{T} \circ \mathrm{e}_{i}=\alpha \circ \mathrm{j}_{i}, i=0,1$. From the universal property of the double mapping cylinder, one obtains that $\mathrm{T} \circ \mathrm{V}=\alpha$.

(3) implies (1) : Let $\mathrm{h}_{0}, \mathrm{~h}_{1}: \mathrm{Y} \rightarrow A, A \in \mathcal{A N} \mathcal{N}$, be given together with a homotopy $\mathrm{H}: \mathrm{X} \times I \rightarrow A$ connecting $\mathrm{h}_{0} \circ \mathrm{f}$ to $\mathrm{h}_{1} \circ \mathrm{f}$. There is a unique $\gamma: D M(\mathrm{f}) \rightarrow A$ such that $\gamma \circ \mathrm{j}_{0}=\mathrm{h}_{0}, \gamma \circ \mathrm{j}_{1}=\mathrm{h}_{1}$ and $\gamma \circ \mathrm{K}=\check{H}$. Since we may write $\mathrm{V}=\mathrm{p}_{\mathrm{v}} \circ \Pi_{\mathrm{V}} \circ \mathrm{e}_{0}$ (see (3.3)), it follows that $\Pi_{\mathrm{V}} \circ \mathrm{e}_{0}$ satisfies (SSE1) too and is a level cofibration. Then there is a $\mathrm{G}: M(\mathrm{~V}) \rightarrow A$ such that $\mathrm{G} \circ \Pi_{\mathrm{V}} \circ \mathrm{e}_{0}=\gamma$. It turns out that $\mathrm{G} \circ \mathrm{j}_{\mathrm{V}}$ is a (global) homotopy connecting $\mathrm{h}^{0}$ and $\mathrm{h}^{1}$. Moreover, one has $\mathrm{G} \circ \mathrm{j}_{\mathrm{v}} \circ(\mathrm{f} \times 1)=$ $\mathrm{G} \circ \mathrm{j}_{\mathrm{v}} \circ \mathrm{V} \circ \mathrm{K}=\gamma \circ \mathrm{K}=\mathrm{H}$.

Corollary 3.7. $\mathrm{V}$ is a shape equivalence whenever $\mathrm{f}$ is a strong shape equivalence and a level cofibration.

Proof. We only have to show that $\mathrm{V}$ induces, for all $A \in \mathcal{A} \mathcal{N} \mathcal{R}$, an onto map $\mathrm{V}_{A}^{*}$ : $[\mathrm{Y} \times I, A] \rightarrow[D M(\mathrm{f}), A]$. Let $\alpha: D M(\mathrm{f}) \rightarrow A$, then $\alpha \circ \mathrm{K}: \mathrm{X} \times I \rightarrow A$ is a homotopy connecting $\alpha \circ \mathrm{j}_{0}$ to $\alpha \circ \mathrm{j}_{1}$. Since $\alpha \circ \mathrm{K} \circ \mathrm{e}_{i}=\alpha \circ \mathrm{j}_{i} \circ \mathrm{f}$, there exists a homotopy $\mathrm{T}: \mathrm{Y} \times I \rightarrow A$, such that $\mathrm{T} \circ(\mathrm{f} \times 1)=\alpha \circ \mathrm{K}$ and $\mathrm{T} \circ \mathrm{e}_{i}=\alpha \circ \mathrm{j}_{i}$. It follows $\mathrm{T} \circ \mathrm{V} \circ \mathrm{K}=$ $\mathrm{T} \circ(\mathrm{f} \times 1)=\alpha \circ \mathrm{K}$ and $\mathrm{T} \circ \mathrm{e}_{i}=\alpha \circ \mathrm{j}_{i}$. From the universal property of the double mapping cylinder, one has $\alpha=\mathrm{T} \circ \mathrm{V}$.

We need to state the following technical result.

Lemma 3.8. Let $\mathrm{f}: \mathrm{X} \rightarrow \mathrm{Y}$ be a strong shape equivalence and a level cofibration in proTop. $\mathrm{f}$ induces a bijection $\mathrm{f}_{\mathcal{D}}^{*}:[\mathrm{Y}, \lim \mathcal{D}] \rightarrow[\mathrm{X}, \lim \mathcal{D}]$, for every finite diagram $\mathcal{D}[\mathbf{1 8}]$ in $\mathcal{A N \mathcal { N }}$, having at most one arrow connecting every two vertices.

Proof. Let $\mathcal{D}$ have vertices $D_{i}, i \in I$, and morphisms $D_{u}: D_{i} \rightarrow D_{j}$, for $u: i \rightarrow j$ in $I$. Assume that $\alpha: \mathrm{X} \rightarrow \lim \mathcal{D}$ is given and let $p_{i}: \lim \mathcal{D} \rightarrow D_{i}, i \in I$, be the projections of the limit. By 3.5(4), for every $i \in I$, there is a promap $\beta_{i}: \mathrm{Y} \rightarrow D_{i}$, such that $\beta_{i} \circ \mathrm{f}=p_{i} \circ \alpha$. If $I(i, j)=\emptyset$, for all $i \in I, i \neq j$, put $\mathrm{h}_{j}=\beta_{j}$. If $u \in I(i, j)$, define $\mathrm{h}_{i}=D_{u} \circ \mathrm{j}$. In this way one obtains a natural cone from $\mathrm{Y}$ to the vertices of the diagram, which induces a unique promap $\mathrm{h}: \mathrm{Y} \rightarrow \lim \mathcal{D}$, with $\mathrm{h} \circ \mathrm{f}=\alpha$.

The proof of the following theorem is partially inspired by Thm. 4.4 of [8].

Theorem 3.9. Let $\mathrm{f}: \mathrm{X} \rightarrow \mathrm{Y}$ be a strong shape equivalence in proTop. Then $\mathrm{f}$ induces a bijection $\mathrm{f}^{*}:[\mathrm{Y}, \mathrm{Z}] \rightarrow[\mathrm{X}, \mathrm{Z}]$, for every strongly fibrant prospace $\mathrm{Z} \in$ $\operatorname{pro} \mathcal{A N} \mathcal{N}$. 
Proof. First of all we may assume, as usual, that $f$ is a level promap with cofinite index set. Moreover, using the mapping cylinder decomposition of $f$, we may also assume that $\mathrm{f}$ is a level cofibration. Let $\mathrm{g}: \mathrm{X} \rightarrow \mathrm{Z}$ be a given promap, with $\mathrm{Z}=$ $\left(Z_{\mu}, z_{\mu \mu^{\prime}}, M\right) \in$ pro $\mathcal{A N} \mathcal{N}$ strongly fibrant. The fact that $\mathrm{f}$ is a shape equivalence, by $3.5(4)$, implies that, for every $\mu \in M$, there is a $\mathrm{k}_{\mu}: \mathrm{Y} \rightarrow Z_{\mu}$ such that $\mathrm{k}_{\mu} \circ \mathrm{f}=\mathrm{g}_{\mu}$. By induction on the number \# $(\mu)$ of the predecessors of $\mu$, let us define $\mathrm{h}_{\mu}=\mathrm{k}_{\mu}$ if $\#(\mu)=0$, and assume to have defined $\mathrm{h}_{\mu}$, for every $\mu \in M$ with $1 \leqslant \#(\mu)<n$, in such a way that $z_{\mu \mu^{\prime}} \circ \mathrm{h}_{\mu^{\prime}}=\mathrm{h}_{\mu}$, for $\mu \leqslant \mu^{\prime}$. Let $\mu^{*} \in M$ having $\#\left(\mu^{*}\right)=n$. The promaps $\mathrm{h}_{\mu}$, for $\mu<\mu^{*}$, define a map $z_{\mu^{*}}: Z_{\mu^{*}} \rightarrow \lim _{\mu \leqslant \mu^{*}} Z_{\mu^{*}}$, and one has $z_{\mu^{*}} \circ \mathrm{k}_{\mu^{*}} \circ \mathrm{f}=z_{\mu^{*}} \circ \mathrm{g}_{\mu^{*}}$. By Lemma 3.8, there is a promap $\gamma: \mathrm{Y} \rightarrow \lim _{\mu \leqslant \mu^{*}} Z_{\mu^{*}}$, with the property that $\gamma \circ \mathrm{f}=z_{\mu^{*}} \circ \mathrm{k}_{\mu^{*}}$. In diagram

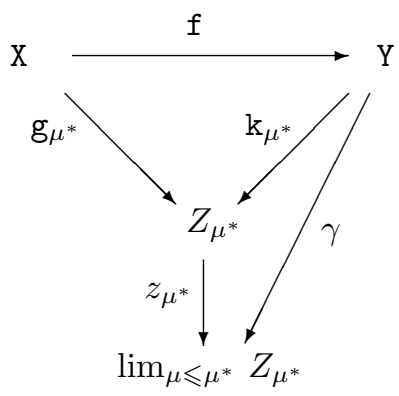

Then, $z_{\mu^{*}} \circ \mathrm{k}_{\mu^{*}} \circ \mathrm{f} \simeq \gamma \circ \mathrm{f}$. Again by Lemma 3.8, since $\mathrm{f}$ is a strong shape equivalence, there is a homotopy $\mathrm{H}: \mathrm{Y} \times I \rightarrow \lim _{\mu \leqslant \mu^{*}} Z_{\mu^{*}}$, with $\mathrm{H}: \gamma \simeq z_{\mu^{*}} \circ \mathrm{k}_{\mu^{*}}$. Since $z_{\mu^{*}}$ is a fibration, there is a homotopy $\mathrm{H}^{*}: \mathrm{Y} \times I \rightarrow Z_{\mu^{*}}$, such that $\mathrm{H}^{*} \circ \mathrm{e}_{0}=\mathrm{k}_{\mu^{*}}$ and $z_{\mu^{*}} \circ \mathrm{H}^{*}=\mathrm{H}$. If we put $\mathrm{h}_{\mu^{*}}=\mathrm{H}^{*} \circ \mathrm{e}_{1}$, the the definition of $\mathrm{h}: \mathrm{Y} \rightarrow \mathrm{Z}$ is complete and one has $\mathrm{h} \circ \mathrm{f}=\mathrm{g}$. Let now $\mathrm{h}, \mathrm{h}^{\prime}: \mathrm{Y} \rightarrow \mathrm{Z}$ be such that $\mathrm{h} \circ \mathrm{f} \simeq \mathrm{h}^{\prime} \circ \mathrm{f}$, by means of a homotopy $\mathrm{F}: \mathrm{X} \times I \rightarrow \mathrm{Z}$. For every $\lambda \in \Lambda, D M\left(f_{\lambda}\right)=X_{\lambda} \times I \cup Y_{\lambda} \times\{0,1\}$ and the inclusion $V_{\lambda}: D M\left(f_{\lambda}\right) \rightarrow Y_{\lambda} \times I$ is a cofibration. If $\tilde{F}_{\lambda}: X_{\lambda} \times I \cup Y_{\lambda} \times\{0,1\} \rightarrow Z_{\lambda}$ is defined by

$$
\tilde{F}_{\lambda}(x, t)= \begin{cases}F_{\lambda}(x, y), & \text { for }(x, t) \in X_{\lambda} \times I \\ h_{\lambda}(x), & t=0 \\ h_{\lambda}^{\prime}(x), & t=1\end{cases}
$$

Then $\tilde{\mathrm{F}}: D M(\mathrm{f}) \rightarrow \mathrm{Z}, \quad \tilde{\mathrm{F}}=\left\{\tilde{F}_{\lambda} \mid \lambda \in \Lambda\right\}$, is a level promap. Since the promap $\mathrm{V}: D M(\mathrm{f}) \rightarrow \mathrm{Y} \times I$ is a shape equivalence and a level cofibration, it follows that $\tilde{\mathrm{F}}$ has an extension $\mathrm{G}: \mathrm{Y} \times I \rightarrow \mathrm{Z}$, which turns out to be a homotopy connecting $\mathrm{h}$ to $\mathrm{h}^{\prime}$.

Theorem 3.10. A continuous map $f: X \rightarrow Y$ is a strong shape equivalence iff it induces a bijection $f^{*}:[Y, Z] \rightarrow[X, Z]$, for every strongly fibered space $Z$.

Proof. Let $f$ be a strong shape equivalence, then by Thm 3.9 it induces a bijection $f^{*}:[Y, \mathrm{Z}] \rightarrow[X, \mathrm{Z}]$, for every strongly fibrant prospace $\mathrm{Z} \in \operatorname{pro} \mathcal{A} \mathcal{N} \mathcal{R}$. Let $Z=\lim \mathrm{Z}$. Since the projection of the limit $\mathrm{p}: Z \rightarrow Z$ induces bijections $[X, Z] \rightarrow[X, Z]$ and 
$[Y, Z] \rightarrow[Y, \mathrm{Z}]$, the first part of the theorem easily follows. Conversely, let $f$ induce bijections $f^{*}:[Y, Z] \rightarrow[X, Z]$, for every strongly fibered space $Z$. Since every ANRspace is strongly fibered, it follows at once that $f$ is a shape equivalence. Taking $\mathrm{Z}=\Phi(X)$, there is a $\mathrm{g}: Y \rightarrow \Phi(X)$ such that $[\mathrm{g} \circ f]=[\mu]$. Since $\mu: X \rightarrow \Phi(X)$ is a strong shape equivalence, it follows that $f$ is such.

Recently, Prasolov [17] has defined the strong shape category of prospaces $s S h$ (proTop) by localizing proTop at the class of strong shape equivalences as defined by the properties (SSE1) and (SSE2) above. The two categories coincide. In fact, from the construction of $s S h(\operatorname{pro} \mathcal{T} o p)$, since ho $(\Phi)$ is reflective, it follows that

$$
\begin{aligned}
& s S h(\operatorname{pro} \mathcal{T} o p) \cong\left(\operatorname{ho}\left(\operatorname{pro} \mathcal{T}_{o p}\right)\right)_{\mathrm{ho}(\Phi)} \cong \operatorname{ho}\left(\operatorname{pro} \mathcal{T}_{o p}\right)\left[\Sigma_{\mathrm{ho}(\Phi)}^{-1}\right] \cong \\
& \cong \operatorname{ho}(\operatorname{pro} \mathcal{T} o p)\left[\Sigma_{s S}^{-1}\right] \cong \operatorname{pro} \mathcal{T}_{o p}\left[\mathcal{S} \mathcal{S} \mathcal{E}^{-1}\right],
\end{aligned}
$$

where $\mathcal{S} \mathcal{E} \mathcal{E}$ is the class of strong shape equivalences in pro $\mathcal{T}_{o p}$, that is those promaps $\mathrm{f} \in$ proTop such that $L(\mathrm{f}) \in \Sigma_{s S}$.

\section{SSDR-promaps.}

In this section we discuss some points from [9] in connection with the results obtained in the previous section. We need some preliminary results before to go on.

Let us recall the following definition from $[\mathbf{9}]$ :

4.1. a promap $\mathrm{f}: \mathrm{X} \rightarrow \mathrm{Y}$ is called an SSDR-promap provided that any commutative diagram in proTop

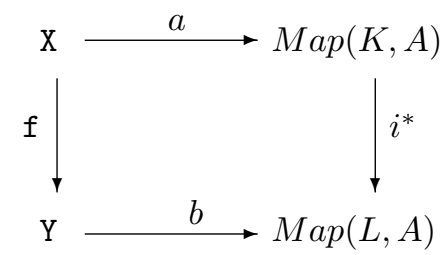

has a filler $\mathrm{Y} \rightarrow \operatorname{Map}(K, A)$, whenever $K$ is a finite $C W$ complex, $L$ is a finite subcomplex, $i: L \rightarrow K$ is the inclusion and $A \in \mathcal{A N \mathcal { N }}$. This notion is a generalization of that of SSDR-map introduced in [4]. $\operatorname{Map}(K, A)$ denotes the space of mappings with the compact-open topology.

In the sequel we shall denote by SSDR the class of SSDR-promaps while $\mathrm{SSDR}_{\mathcal{T}_{o p}}$ will be the subclass of SSDR whose elements are of the form $\mathrm{f}: \mathrm{X} \rightarrow Y, Y \in \mathcal{T}$ op.

Thm. 3.5 of $[\mathbf{9}]$ states that $\mathrm{f}: \mathrm{X} \rightarrow \mathrm{Y}$ is an SSDR promap iff it satisfies the following two conditions :

(SSDR1) for every $A \in \mathcal{A N \mathcal { R }}$ and for every $\mathrm{h}: \mathrm{X} \rightarrow A$, there is a $\mathrm{g}: \mathrm{Y} \rightarrow A$, such that $\mathrm{g} \circ \mathrm{f}=\mathrm{h}$,

(SSDR2) given morphisms $\mathrm{g}, \mathrm{h}: \mathrm{Y} \rightarrow A, A \in \mathcal{A N \mathcal { N }}$, and a global homotopy $\mathrm{F}: \mathrm{X} \times I \rightarrow A$ joining $\mathrm{f} \circ \mathrm{g}$ and $\mathrm{f} \circ \mathrm{h}$, there exists a global homotopy $\mathrm{G}: \mathrm{Y} \times I \rightarrow A$ joining $\mathrm{g}$ and $\mathrm{h}$, such that $\mathrm{F}=\mathrm{G} \circ(\mathrm{f} \times 1)$. 


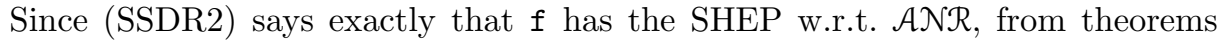
$3.5(4)$ and 3.6, one obtains the

Theorem 4.2. TheOrem 4.2 Let $\mathrm{f}: \mathrm{X} \rightarrow \mathrm{Y}$ be a level cofibration in proTop. $\mathrm{f}$ is an SSDR promap iff it is a strong shape equivalence.

Remark 4.3. As a consequence of the theorem, it follows that every trivial cofibration is an SSDR-promap. In fact, by ([10], 3.3.36), one may assume, up to isomorphisms, that $f$ is a level trivial cofibration. Then, it is clear that every (strongly) SSDR-fibrant prospace is also (strongly) fibrant. Moreover, if $\mathrm{Z}$ is a (strongly) SSDR-fibered prospace, then its inverse limit lim Z is $\mathrm{SSDR}_{\mathcal{T}}$ - -fibrant: let $\mathrm{f}: \mathrm{X} \rightarrow Y \in \mathrm{SSDR}_{\mathcal{T} o p}$ and $\mathrm{a}: \mathrm{X} \rightarrow \lim \mathrm{Z}$, be given. If $\mathrm{p}: \lim \mathrm{Z} \rightarrow \mathrm{Z}$ is the limiting

cone, there is a $\mathrm{y}: Y \rightarrow \mathrm{Z}$, such that $\mathrm{y} \circ \mathrm{f}=\mathrm{p} \circ$ a and, by the universal property of the limit, there is also a $\mathrm{t}: Y \rightarrow \lim \mathrm{Z}$, with $\mathrm{p} \circ \mathrm{t}=\mathrm{y}$. It follows that $\mathrm{t} \circ \mathrm{f}=\mathrm{a}$.

\section{References}

[1] R.A. Alò - H.L. Shapiro, Normal topological spaces, C.U.P., 1974.

[2] K. Brown, Abstract homotopy theories and generalized sheaf cohomology, Trans. Amer. Math. Soc. 186 (1973), 419-455.

[3] R. Brown, Topology, Ellis Horwood, 1988.

[4] F.W. Cathey, Strong shape theory, Shape Theory and Geometric Topology, Proc. Dubrovnik 1981, Lecture Notes in Math. 870, Springer, 1981, 215-238.

[5] F.W. Cathey - J. Segal, Strong shape theory and resolutions, Top. Appl. 15 (1983), 119-130.

[6] A. Calder - H. M. Hastings, Realizing strong shape equivalences, J. Pure Appl. Alg. 20 (1981), 129-156.

[7] J.M. Cordier - T. Porter, Categorical shape theory, World Scientific, 1996.

[8] J. Dydak - S. Nowak, Strong shape for topological spaces, Trans. Amer. Math. Soc. 323 (2) (1991), 765-796.

[9] J. Dydak - S. Nowak, Function spaces and shape theories, preprint.

[10] D. A. Edwards - H. M. Hastings, Čech and Steenrod homotopy theories, Lectures Notes in Math. 542, Springer, 1976.

[11] P.J. Hilton, Homotopy Theory and Duality, Gordon Breach, 1965.

[12] K.H. Kamps - T. Porter, Abstract Homotopy and Simple Homotopy Theory, World Scientific, 1997.

[13] S. Mardešić, Approximate polyhedra, resolutions of maps and shape fibrations, Fund. Math. 114 (1981), 53-78.

[14] S. Mardešić, Strong shape and homology, Springer Verlag, 2000.

[15] S. Mardešić - J. Segal, Shape theory, North Holland, 1982.

[16] T. Porter, On the two definitions of Ho(Pro C), Top. Appl. 28 (1988), 289293. 
[17] A.V. Prasolov, Extraordinary strong homology, Top. Appl. 113 (2001), 249291.

[18] H. Schubert, Categories, Springer-Verlag, 1974.

[19] L. Stramaccia, On the definition of the strong shape category, Glasnik Mat. 32 (52) (1997), 141-151.

[20] L. Stramaccia, Characterizing shape theories by Kan extensions, Top. Appl. 120 (2002), 355-363.

[21] L. Stramaccia, Shape and strong shape equivalences, Cahiers Topologie Géom. Différentielle Catég. (to appear)

[22] W. Tholen, Pro-categories and multiadjoint functors, Can. J. Math. 36 (1984), 144-155.

This article may be accessed via WWW at http://www.rmi.acnet.ge/hha/ or by anonymous ftp at

ftp://ftp.rmi.acnet.ge/pub/hha/volumes/2002/n1a6/v4n1a6.(dvi,ps,pdf)

L. Stramaccia stra@dipmat.unipg.it

Dipartimento di Matematica e Informatica

Universitá di Perugia

via Vanvitelli, I-06123 Perugia 